Appeared in Advanced Robotics, 10 (6), 1996: 547-578.

\title{
ON THE DESIGN OF BEHAVIOR-BASED MULTI-ROBOT TEAMS
}

Lynne E. Parker

Center for Engineering Systems Advanced Research, Oak Ridge National Laboratory, P. O. Box 2008, Mailstop 6364, Oak Ridge, TN 37831-6364 USA

\begin{abstract}
Real-world applications that are ideal for robotic solutions are very complex and challenging. Many of these applications are set in dynamic environments that require capabilities distributed in functionality, space, or time. These applications, therefore, often require teams of robots to work together cooperatively to successfully address the mission. While much research in recent years has addressed the issues of autonomous robots and multi-robot cooperation, current robotics technology is still far from achieving many of these real world applications. We believe that two primary reasons for this technology gap are that (1) previous work has not adequately addressed the issues of fault tolerance and adaptivity in multi-robot teams, and (2) existing robotics research is often geared at specific applications, and is not easily generalized to different, but related, applications. This paper addresses these issues by first describing the design issues of key importance in these real-world cooperative robotics applications - fault tolerance, reliability, adaptivity, and coherence. We then present a general architecture addressing these design issues - called ALLIANCE - that facilitates multi-robot cooperation of small- to mediumsized teams in dynamic environments, performing missions composed of loosely coupled subtasks. We illustrate the generality of this architecture by describing two very different proof-of-concept implementations of this architecture: a janitorial service mission, and a bounding overwatch mission.
\end{abstract}

Key words: Multi-robot team design; cooperative robotics; ALLIANCE; control architectures; behavior-based systems

\section{INTRODUCTION}

A key driving force in the development of mobile robotic systems is their potential for reducing the need for human presence in dangerous applications, such as the cleanup of toxic waste, nuclear power plant decommissioning, planetary exploration, search and rescue missions, security, surveillance, and reconnaissance tasks; or in repetitive types of tasks, such as automated manufacturing or industrial/household maintenance. The nature of many of these challenging work environments requires the robotic systems to work fully autonomously in achieving human-supplied goals. One approach to designing these autonomous systems is to develop a single robot that can accomplish particular goals in a 
given environment. However, the complexity of many environments or missions may require a mixture of robotic capabilities that is too extensive to design into a single robot. Additionally, time constraints may require the use of multiple robots working simultaneously on different aspects of the mission in order to successfully accomplish the objective. In some instances, it may actually be easier or cheaper to design cooperative teams of robots to perform some mission than it would be to use a single robot. Thus, we must build teams of possibly heterogeneous robots that can work together to accomplish a mission that no individual robot can accomplish alone.

Although a significant amount of research on mobile robotics has been accomplished in the last two decades, relatively little of this research has been transferred to use in real-world applications. We believe that two primary reasons for this are that: (1) previous research has not adequately addressed the problems inherent in real-world applications (such as robot failures and dynamic environments) and (2) existing robotics research is often geared at specific applications, and uses concepts not easily generalized to different, but related, applications. This paper addresses these issues by first describing the design goals that must be met for real-world robotic applications. We present a general behavior-based architecture, called ALLIANCE, that was designed with these goals in mind, resulting in a fault tolerant, reliable, adaptive, and coherent mechanism for cooperative robot control. We then illustrate the generality of ALLIANCE by presenting proof-of-principle implementations in two quite different application domains - janitorial service and bounding overwatch. We conclude with a discussion of how ALLIANCE meets our design goals.

The next section presents the design requirements that must be met in a successful cooperative robot approach. Section 3 describes related work in cooperative mobile robotics. Section 4 presents the ALLIANCE architecture that was designed to meet the requirements of robustness, reliability, flexibility, and coherence. Section 5 illustrates the generality of ALLIANCE by presenting two proof-of-concept implementations of the ALLIANCE architecture: the janitorial service and the bounding overwatch missions. Section 6 compares the $a$ priori design requirements with the design of the ALLIANCE architecture. We conclude the paper in section 7 with some closing remarks on the design of cooperative robot teams.

\section{DESIGN REQUIREMENTS OF A COOPERATIVE ARCHITECTURE}

The difficulties in designing a cooperative team are significant. In [4], Bond and Gasser describe the basic problems the field of Distributed Artificial Intelligence must address; those aspects directly related to situated multi-robot systems include the following:

- How do we formulate, describe, decompose, and allocate problems among 
a group of intelligent agents?

- How do we enable agents to communicate and interact?

- How do we ensure that agents act coherently in their actions?

- How do we allow agents to recognize and reconcile conflicts?

The ALLIANCE architecture described in this paper offers one solution to the above questions. In addition to answering these questions, however, one of our primary design goals in developing a multi-robot cooperative architecture is to allow the resulting robotic teams to be robust, reliable, and flexible. The following subsections discuss these design requirements that we feel are particularly important in the design of cooperative robotics teams.

\subsection{Robustness and Fault Tolerance}

Robustness refers to the ability of a system to gracefully degrade in the presence of partial system failure; the related notion of fault tolerance refers to the ability of a system to detect and compensate for partial system failures. Requiring robustness and fault tolerance in a cooperative architecture emphasizes the need to build cooperative teams that minimize their vulnerability to individual robot outages - a requirement that has many implications for the design of the cooperative team.

To achieve this design requirement, one must first ensure that critical control behaviors are distributed across as many robots as possible rather than being centralized in one or a few robots. This complicates the issue of action selection among the robots, but results in a more robust multi-robot team since the failure of one robot does not jeopardize the entire mission.

Second, one must ensure that an individual robot does not rely on orders from a higher-level robot to determine the appropriate actions it should employ. Relying on one, or a few, coordinating robots makes the team much more vulnerable to individual robot failures. Instead, each robot should be able to perform some meaningful task, up to its physical limitations, even when all other robots have failed.

And third, one must ensure that robots have some means for redistributing tasks among themselves when robots fail. This characteristic of task reallocation is essential for a team to accomplish its mission in a dynamic environment.

\subsection{Reliability}

Reliability refers to the dependability of a system, and whether it functions properly each time it is utilized. To properly analyze a cooperative robot architecture, one should separate the architecture itself from the robots on which 
the architecture is implemented. Clearly, if the architecture is implemented on robots that only function a small percentage of the time, one cannot expect a very dependable result from trial to trial. One measure of the reliability of the architecture is its ability to guarantee that the mission will be solved, within certain operating constraints, when applied to any given cooperative robot team. Without this characteristic, the usefulness of a control architecture is clearly much diminished.

As an example of a reliability problem exhibited in a control architecture, consider a situation in which two robots, $r_{1}$ and $r_{2}$, have two tasks, $t_{1}$ and $t_{2}$, to perform. Let us assume that their control architecture leads them to negotiate a task allocation which results in $r_{1}$ performing task $t_{1}$ and $r_{2}$ performing task $t_{2}$. Further suppose that $r_{1}$ experiences a mechanical failure that neither $r_{1}$ nor $r_{2}$ can detect. While $r_{1}$ continues to attempt to complete task $t_{1}$, robot $r_{2}$ successfully completes task $t_{2}$. However, although $r_{2}$ also has the ability to successfully complete task $t_{1}$, it does nothing further because it knows that $r_{1}$ is performing that task. Thus, the robots continue forever, never completing the mission. One would not term such a control architecture reliable, since a mere reallocation of the tasks would have resulted in the mission being successfully completed.

\subsection{Flexibility and Adaptivity}

The terms flexibility and adaptivity refer to the ability of team members to modify their actions as the environment or robot team changes. Ideally, the cooperative team should be responsive to changes in individual robot skills and performance as well as dynamic environmental changes. This requirement reflects the fact that the capabilities of robots may change over time due to learning, which should enhance performance, or due to mechanical or environmental causes that may reduce or increase a robot's success at certain tasks. Team members should respond to these changes in performance by taking over tasks that are no longer being adequately performed or by relinquishing those tasks better executed by others. Each robot must decide which task it will undertake based on the actual performance of tasks by other robots, rather than on what other robots say that they are able to accomplish.

Robots must also exhibit flexibility in their action selection during the mission in response to the dynamic nature of their environment. Obviously, in real environments changes occur that cannot be attributed to the actions of any robot team member or members. Rather, outside forces not under the influence of the robot team affect the state of the environment throughout the mission. These effects may be either destructive or beneficial, leading to an increase or decrease in the workload of the robot team members. The robot team should therefore be flexible in its action selections, opportunistically adapting to environmental changes that eliminate the need for certain tasks, or activating other tasks that a new environmental state requires. 


\subsection{Coherence}

Coherence refers to how well the team performs as a whole, and whether the actions of individual agents combine toward some unifying goal. Typically, coherence is measured along some dimension of evaluation, such as the quality of the solution or the efficiency of the solution [4]. Efficiency considerations are particularly important in teams of heterogeneous robots whose capabilities overlap, since different robots are often able to perform the same task, but with quite different performance characteristics. To obtain a highly efficient team, the control architecture should ensure that robots select tasks such that the overall mission performance is as close to optimal as possible.

A team in which agents pursue incompatible actions, or in which they duplicate each other's actions cannot be considered a highly coherent team. On the other hand, designing a coherent team does not require the elimination of all possible conflict. Rather, the agents must be provided with some mechanism to resolve the conflicts as they arise. A simple example of conflict occurs whenever multiple robots share the same workspace; although they may have the same high-level goals, they may at times try to occupy the same position in space, thus requiring them to resolve their positioning conflict. This can usually be accomplished through a very simple protocol.

Clearly, multi-robot teams exhibiting low coherence are of limited usefulness in solving practical engineering problems. A design goal in building cooperative robot teams must therefore be to achieve high coherence.

\section{RELATED WORK}

A significant body of research in cooperative mobile robotics deals with the study of large numbers (often called swarms) of homogeneous robots. This approach to multi-robot cooperation is useful for non-time-critical applications involving numerous repetitions of the same activity over a relatively large area, such a cleaning a parking lot or collecting rock samples on Mars. The approach to cooperative control taken in these systems is derived from the fields of neurobiology, ethology, psychophysics, and sociology, and is typically characterized by teams of large numbers of homogeneous robots, each of which has fairly limited capabilities on its own. However, when many such simple robots are brought together, globally interesting behavior can emerge as a result of the local interactions of the robots. A key research issue in this scenario is determining the proper design of the local control laws that will allow the collection of robots to solve a given problem.

A number of researchers have studied the issues of swarm robotics. Deneubourg et al. [9] describe simulation results of a distributed sorting algorithm. Theraulaz et al. [28] extract cooperative control strategies, such as foraging, from a study of Polistes wasp colonies. Steels [26] presents simulation studies of the use of several dynamical systems to achieve emergent functionality as applied to 
the problem of collecting rock samples on a distant planet. Drogoul and Ferber [10] describe simulation studies of foraging and chain-making robots. In [16] Mataric describes the results of implementing group behaviors such as dispersion, aggregation, and flocking on a group of physical robots. Beni and Wang [3] describe methods of generating arbitrary patterns in cyclic cellular robotics. Kube and Zhang [14] present the results of implementing an emergent control strategy on a group of five physical robots performing the task of locating and pushing a brightly lit box. Stilwell and Bay [27] present a method for controlling a swarm of robots using local force sensors to solve the problem of the collective transport of a palletized load. Arkin et al. [1] present research concerned with sensing, communication, and social organization for tasks such as foraging. Ichikawa and Hara [12] investigate the emergent ability of swarm intelligence as applied to a space coverage problem. The CEBOT work, described in [11] and many related papers, has many similar goals to other swarm-type multi-robotic systems, although the CEBOT robots can be one of a number of robot classes, rather than purely homogeneous.

Another primary area of research in cooperative control deals with achieving "intentional" cooperation among a limited number of typically heterogeneous robots performing several distinct tasks. In this type of cooperative system, the robots often have to deal with an efficiency constraint that requires a more directed type of cooperation than is found in the swarm approach described above. Although individual robots in this approach are typically able to perform useful tasks on their own, groups of such robots are often able to accomplish missions that no individual robot can accomplish on its own. The general research issues of adaptive action selection, communication, and conflict resolution are of particular importance in these types of systems. The types of applications addressed in the current paper fall into this second category - "intentional" cooperation.

Two bodies of previous research are particularly applicable to this second type of cooperation. First, several researchers have directly addressed this cooperative robot problem by developing control algorithms and implementing them either on physical robots or on simulations of physical robots that make reasonable assumptions about robot capabilities. Examples of this research include the work of Lueth and Laengle [15] who study the issue of fault-tolerant behavior and error recovery in a distributed control architecture called KAMARA; Noreils [17], who proposes a three-layered control architecture that includes a planner level, a control level, and a functional level; Caloud et al. [7], who describe an architecture that includes a task planner, a task allocator, a motion planner, and an execution monitor; Asama et al. [2] who describe an architecture called ACTRESS that utilizes a negotiation framework to allow robots to recruit help when needed; Cohen et al. [8], who use a hierarchical division of authority to address the problem of cooperative fire-fighting; Ota et al. [18], who describe a mechanism for robot selection of strategies via several tactics; and Wang [29], who proposes the use of several distributed mutual exclusion 
algorithms that use a "sign-board" for inter-robot communication.

The second, significantly larger, body of research related to intentional cooperation comes from the Distributed Artificial Intelligence (DAI) community, which has produced a great deal of work addressing this type of intentional cooperation among generic agents. These agents are typically software systems running as interacting processes to solve a common problem rather than embodied, sensor-based robots. In most of this work, the issue of task allocation has been the driving influence that dictates the design of the architecture for cooperation. Typically, the DAI approaches use a distributed, negotiation-based mechanism to determine the allocation of tasks to agents. See [4] for many of the seminal papers in this field.

\section{THE ALLIANCE ARCHITECTURE}

In this section, after describing our intended application domain, we describe a control architecture - called ALLIANCE - that was designed with the above design requirements in mind. Reasons for the design selections we made will be noted in this section, then summarized and compared to our design requirements later in the paper.

\subsection{ALLIANCE Application Domain}

ALLIANCE has been designed for multi-robot missions with the following characteristics:

- Mission tasks are distributed either spatially, functionally, or temporally

- Mission tasks are loosely-coupled and independent (except that they may have fixed ordering dependencies)

- Broadcast communication is usually available (i.e., it may be noisy or fail at times)

- Robot teams are small- or medium-sized (i.e., up to tens, rather than hundreds or thousands of robots)

In addition, ALLIANCE is particularly well-suited for missions involving the following situations that we believe are true for most real-world applications of cooperative robotics:

- Dynamic environments

- Dynamic team composition (e.g., due to robot failures or to new robots being added to the team)

- The team is composed of heterogeneous robots with overlapping capabilities. 


\subsection{ALLIANCE Mechanisms}

ALLIANCE is a fully distributed architecture for fault tolerant, heterogeneous robot cooperation that utilizes adaptive action selection to achieve cooperative control for small- to medium-sized mobile robot teams. Under this architecture, the robots possess a variety of high-level task-achieving functions that they can perform during a mission, and must at all times select an appropriate action based on the requirements of the mission, the activities of other robots, the current environmental conditions, and their own internal states. The missions that the robot team can address under ALLIANCE are restricted to those missions which are composed of loosely coupled subtasks that are independent, but may have fixed ordering dependencies among them. We note, however, that even with this restriction, the proposed architecture covers a very large range of missions for which cooperative robots are useful.

In ALLIANCE, individual robots are designed using a behavior-based approach [5], in order to achieve robustness at the individual robot level. Under the behavior-based construction, a number of task-achieving behaviors are active simultaneously, each receiving sensory input and controlling some aspect of the actuator output. The lower-level behaviors, or competences, correspond to primitive survival behaviors such as obstacle avoidance, while the higher-level behaviors correspond to higher goals such as map building and exploring. The output of the lower-level behaviors can be suppressed or inhibited by the upper layers when the upper layers deem it necessary. This approach has been used successfully in a number of robotic applications, several of which are described in [6].

Extensions to this approach are necessary, however, when a robot must select among a number of competing actions - actions which cannot be pursued in parallel. Unlike typical behavior-based approaches, ALLIANCE delineates several behavior sets that are either active as a group or hibernating. Figure 1 shows the general architecture of ALLIANCE and illustrates three such behavior sets. The $j$ th behavior set, $a_{i j}$, of a robot $r_{i}$ corresponds to those levels of competence required to perform some high-level task-achieving function. When a robot activates a behavior set, we say that it has selected the task corresponding to that behavior set. Since different robots may have different ways of performing the same task, and therefore activate different behavior sets to perform that task, we define the function $h_{i}\left(a_{i j}\right)$, for all robots $r_{i}$ on the team, to refer to the task that robot $r_{i}$ is working on when it activates its $j$-th behavior set, $a_{i j}$.

Because of the alternative goals that may be pursued by the robots, the robots must have some means of selecting the appropriate behavior set to activate. Thus, controlling the activation of each of these behavior sets is a motivational behavior. Due to conflicting goals, only one behavior set per robot can be active at any point in time. This restriction is implemented via cross-inhibition of motivational behaviors, represented by the arcs at the top of Figure 1, in which the activation of one behavior set suppresses the activation of all other behavior 
The ALLIANCE Architecture

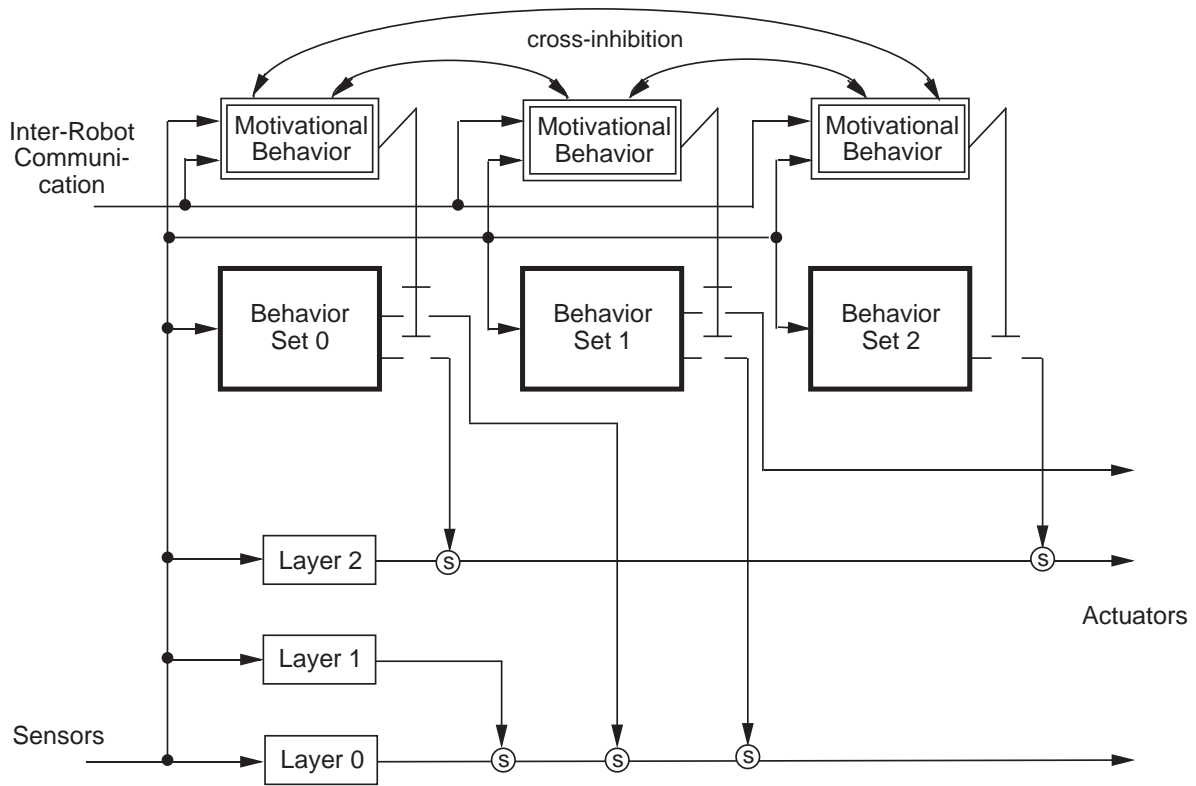

Figure 1: The ALLIANCE architecture. The symbols in this figure that connect the output of each motivational behavior with the output of its corresponding behavior set (vertical lines with short horizontal bars) indicate that a motivational behavior either allows all or none of the outputs of its behavior set to pass through to the robot's actuators.

sets. However, other lower-level competences such as collision avoidance may be continually active regardless of the high-level goal the robot is currently pursuing. Examples of this type of continually active competence are shown in Figure 1 as layer 0 , layer 1 , and layer 2 .

The primary mechanism for achieving adaptive action selection in this architecture is the motivational behavior. At all times during the mission, each motivational behavior receives input from a number of sources, including sensory feedback, inter-robot communication, inhibitory feedback from other active behaviors, and internal motivations called robot impatience and robot acquiescence. The output of a motivational behavior is the activation level of its corresponding behavior set, represented as a non-negative number. When this activation level exceeds a given threshold, the corresponding behavior set becomes active.

Intuitively, a motivational behavior works as follows. Robot $r_{i}$ 's motivation to activate any given behavior set $a_{i j}$ is initialized to 0 . Then, over time, robot $r_{i}$ 's motivation $m_{i j}(t)$ to activate behavior set $a_{i j}$ increases at a "fast" rate (called $\left.\delta_{-} f_{a s t} t_{i j}(t)\right)$ as long as the task corresponding to that behavior set (i.e. $\left.h_{i}\left(a_{i j}\right)\right)$ 
is not being accomplished, as determined from sensory feedback. However, the robots must be responsive to the actions of other robots, adapting their task selection to the activities of team members. Thus, if a robot $r_{i}$ is aware that another robot $r_{k}$ is working on task $h_{i}\left(a_{i j}\right)$, then $r_{i}$ is satisfied for some period of time that the task is going to be accomplished even without its own participation, and thus go on to some other applicable action. Its motivation to activate behavior set $a_{i j}$ still increases, but at a slower rate (called $\left.\delta_{-} s l o w_{i j}(k, t)\right)$. This characteristic prevents robots from replicating each other's actions and thus wasting needless energy. Of course, detecting and interpreting the actions of other robots (often called action recognition) is not a trivial problem, and often requires perceptual abilities that are not yet possible with current sensing technology. As it stands today, the sensory capabilities of even the lower animals far exceed present robotic capabilities. Thus, to enhance the robots' perceptual abilities, ALLIANCE utilizes a simple form of broadcast communication to allow robots to inform other team members of their current activities, rather than relying totally on sensory capabilities. At some pre-specified rate, each robot $r_{i}$ broadcasts a statement of its current action, which other robots may listen to or ignore as they wish. No two-way conversations are employed in this architecture. Since we have designed the architecture for small- to medium-sized robot teams (i.e. tens of robots rather than hundreds or thousands), the issue of the communication cost for large numbers of robots is avoided. This broadcast form of communication is not designed for applications involving large "swarm"-type robot teams.

Each robot is designed to be somewhat impatient, however, in that a robot $r_{i}$ is only willing for a certain period of time to allow the communicated messages of another robot to affect its own motivation to activate a given behavior set. Continued sensory feedback indicating that a task is not getting accomplished thus overrides the statements of another robot that it is performing that task. This characteristic allows robots to adapt to failures of other robots, causing them to ignore the activities of a robot that is not successfully completing its task.

A complementary characteristic in these robots is that of acquiescence. Just as the impatience characteristic reflects the fact that other robots may fail, the acquiescence characteristic indicates the recognition that a robot itself may fail. This feature operates as follows. As a robot $r_{i}$ performs a task, its willingness to give up that task increases over time as long as the sensory feedback indicates the task is not being accomplished. As soon as some other robot $r_{k}$ indicates it has begun that same task and $r_{i}$ feels it (i.e. $r_{i}$ ) has attempted the task for an adequate period of time, the unsuccessful robot $r_{i}$ gives up its task in an attempt to find an action at which it is more productive. Additionally, even if another robot $r_{k}$ has not taken over the task, robot $r_{i}$ may give up its task anyway if it is not completed in an acceptable period of time. This allows $r_{i}$ the possibility of working on another task that may prove to be more productive rather than becoming stuck performing the unproductive task forever. With 
this acquiescence characteristic a robot is able to adapt its actions to its own failures.

The behavior-based design of the motivational behaviors also allows the robots to adapt to unexpected environmental changes which alter the sensory feedback. The need for additional tasks can suddenly occur, requiring the robots to perform additional work, or existing environmental conditions can disappear and thus relieve the robots of certain tasks. In either case, the motivations fluidly adapt to these situations, causing robots to respond appropriately to the current environmental circumstances. Refer to [19, 25] for more mathematical details of the ALLIANCE architecture, including the formal mathematical model of ALLIANCE, proofs of correction which guarantee that ALLIANCE will allow the robot team to accomplish its mission under certain conditions, and results of physical robot implementations of the ALLIANCE architecture for a mock toxic waste cleanup mission and a box-pushing mission.

\section{TWO IMPLEMENTATIONS OF ALLIANCE}

The ALLIANCE architecture has been successfully implemented in a variety of proof-of-concept applications on both physical and simulated mobile robots. The applications implemented on physical robots include a laboratory version of hazardous waste cleanup [20, 21, 22] and a cooperative box pushing demonstration [19, 22] The applications using simulated mobile robots include a janitorial service mission and a bounding overwatch mission (reminiscent of military surveillance). All of these missions using the ALLIANCE architecture have been well-tested. Over 50 logged physical robot runs of the hazardous waste cleanup mission and over 30 physical robot runs of the box pushing demonstration were completed to elucidate the important issues in heterogeneous robot cooperation. Many runs of each of these physical robot applications are available on videotape. The missions implemented on simulated robots (including those reported in this paper) encompass hundreds of runs each.

After briefly describing the multi-robot simulator used in these implementations, we present the design and results of implementation of the ALLIANCE architecture in the janitorial service and bounding overwatch missions. We present these designs in some detail, to illustrate the generality of ALLIANCE, and to facilitate the further use of ALLIANCE in real-world applications.

Note that during the design of any implementation of ALLIANCE, it is necessary for the human to determine the values of the parameters of the system that correspond to the rates of impatience and acquiescence of each of the motivations in a robot, the rate at which robots will broadcast their current actions to their teammates, and the thresholds at which the behaviors will activate. The rates of impatience and acquiescence are interrelated to the motivational threshold, in that varying the threshold at which a behavior set activates has the same effect as varying the rates of impatience/acquiescence with a fixed threshold. Thus, in our experiments, we held the threshold value constant across all 
behaviors of all robots, and initiated the rates of impatience/acquiescence based upon experimental trials of the robots performing similar missions.

Some care has to be taken in setting the parameters, since inordinately high rates of impatience and acquiescence can cause the robots to "thrash" back and forth between tasks, exhibiting very short attention spans, while inordinately slow rates of impatience/acquiescence can make the robots waste large periods of time. However, in practice, we have found that determining the proper parameter settings is not difficult. The ALLIANCE architecture has been implemented on a number of quite different robotic applications, and parameter tuning did not prove to be a problem. Ideally, however, the robots on the cooperative team should be able to adapt these values with experience to find the right parameter settings that moderate between the two extremes, rather than relying on human tuning. We have developed a learning system called L-ALLIANCE that allows the robots to adapt these parameters during a mission, and from mission to mission, based upon robot experience and the composition and capabilities of the robot team. For example, if robot capabilities change over time due to learning or mechanical problems, or if environmental changes affect the robot team's performance the robots are able to compensate by automatically updating their parameters. Further details of the L-ALLIANCE mechanism are beyond the scope of this paper, but are available in [24].

\subsection{The Multi-Robot Simulator}

The original version of our cooperative robot simulator was developed in the MIT Mobot Laboratory by Yasuo Kagawa, a visiting scientist from the Mazda Corporation. The author modified the internal mechanisms of the original version extensively, however, to improve its response time and lower its memory requirements significantly, and to increase the available sensory and effector models. This simulator, written in object-oriented Allegro Common Lisp, runs in the Macintosh environment and is designed to simulate indoor office-type environments. The simulator provides most of the features one would expect in such a cooperative robot simulator, including the ability to create a simulated office environment with obstacles and walls, to define sensory and effector devices, to define robots possessing any given combination of sensors and effectors, and to allow robots to communicate with each other.

The sensors that have been developed for the janitorial service and bounding overwatch missions are a ranging sensor, an infrared beacon detector, a compass, a microphone, an $x, y$ positioning system, a pyroelectric sensor, and a dust sensor (see [30] for an example of a real-life dust sensor). Each robot's locomotion is commanded by velocity and turn angle values; additional effectors implemented are a floor vacuum, a garbage dumper, and a duster arm that can be extended either right or left. Since our purpose for the multi-robot simulator was to test and demonstrate cooperative control issues, rather than new mechanical devices, we did not mechanically model these effectors; they merely act as switches to 
turn some effect on or off, based on the robot's current position and its proximity to objects of certain types. To incorporate uncertainty into the system, we added $20 \%$ random noise to the sensors and effectors.

Obstacle objects in the simulator can be of two types - convex polyhedral objects and one-dimensional wall-type objects. These objects can possess a number of additional characteristics, such as the ability to emit an IR beacon or sound at a specified intensity, or to possess a certain amount of dust, garbage, or heat. An additional feature of the simulator is that objects can be moved manually during the robot simulation, thus allowing the experimenter to mimic a dynamic environment.

\subsection{The Janitorial Service Mission}

The janitorial service mission requires a robot team to clean a room in an office building that is unfamiliar to the robots at the beginning of the task, and may change dynamically due to people occupying the room. Figure 2 shows a typical environment used in these simulations. The overall mission consists of three high-level tasks: emptying the garbage, dusting the furniture, and cleaning the floors. The robots assigned to this mission are not familiar with the capabilities of the other robots in the team, which may change dynamically due to mechanical failure or environmental changes. Each robot has a different mixture of capabilities which allows it to complete a portion of the mission on its own. However, since no single robot has all the capabilities necessary to accomplish the mission alone, the robots must work as a team. Due to limited time and energy, the robots must accomplish their mission efficiently, minimizing redundant actions as much as possible. The efficiency requirements and the dynamic environment of this mission require the robots to adapt their activities over time due to the current and previous actions of other robots, and to the sensory feedback received from the environment.

This mission offers the opportunity to illustrate methods of implementing the ALLIANCE architecture for missions involving numerous independent repetitions of the same subtask. For example, although this mission is composed of three high-level tasks - emptying the garbage, dusting the furniture, and cleaning the floor - each of these tasks involves a number of separate subtasks which could be performed independently by more than one robot, such as having different robots dust different pieces of furniture simultaneously. We illustrate some techniques for allowing one motivational behavior to control which subtask a given robot elects to perform. A slightly different method of this task subdivision is used in this mission for each of the three high-level tasks.

5.2.1. Robot Software Design. The ALLIANCE architecture specifies that the team's mission be segmented into distinct high-level tasks that must be accomplished. The janitorial service mission can be segmented into three such tasks - empty-garbage, dust-furniture, and clean-floor. Each of these tasks is thus designed as a separate behavior set. Figure 3 shows the software control of a 


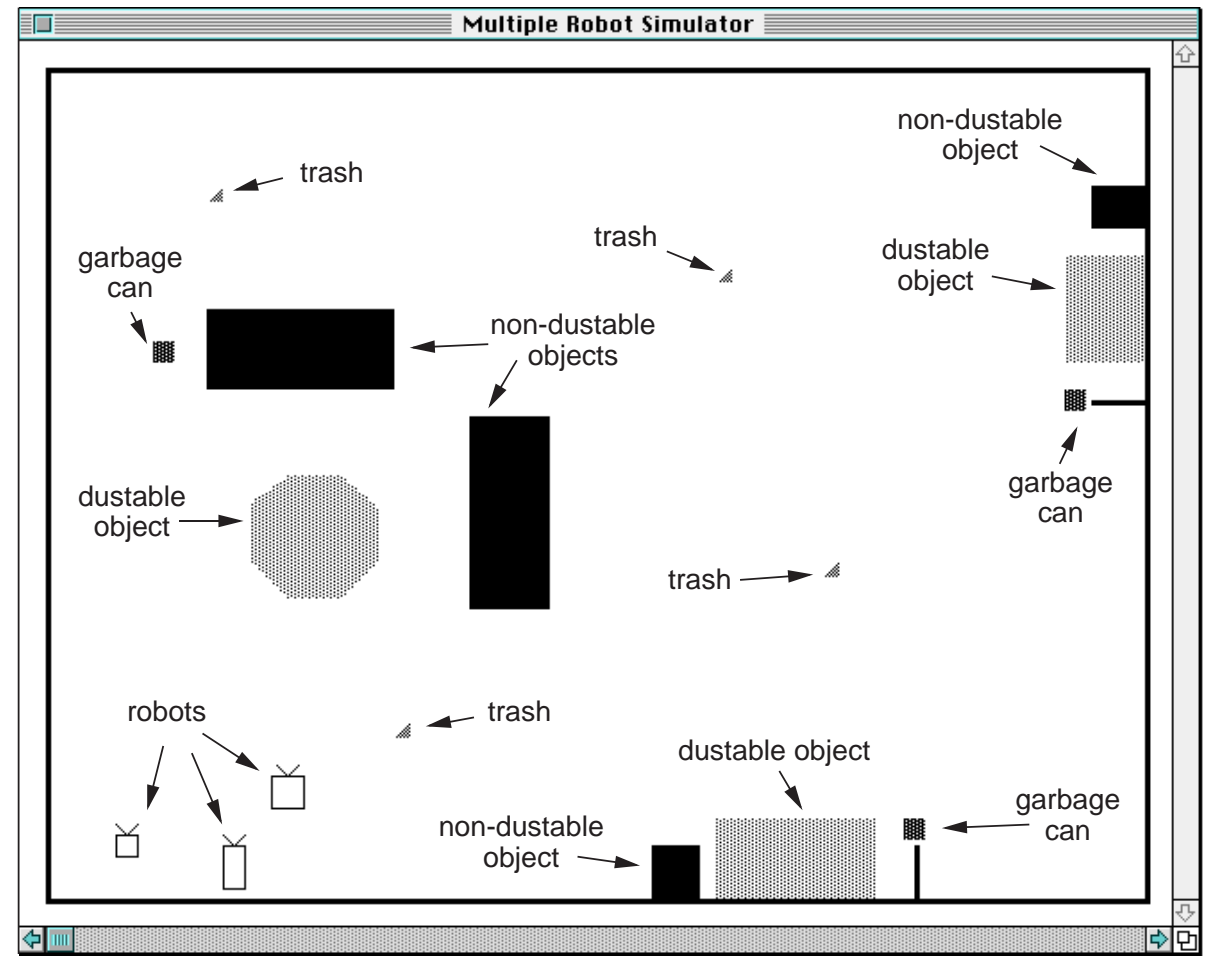

Figure 2: Labeled objects in a typical janitorial service experiment. 
Janitorial Service: Behavior Organization

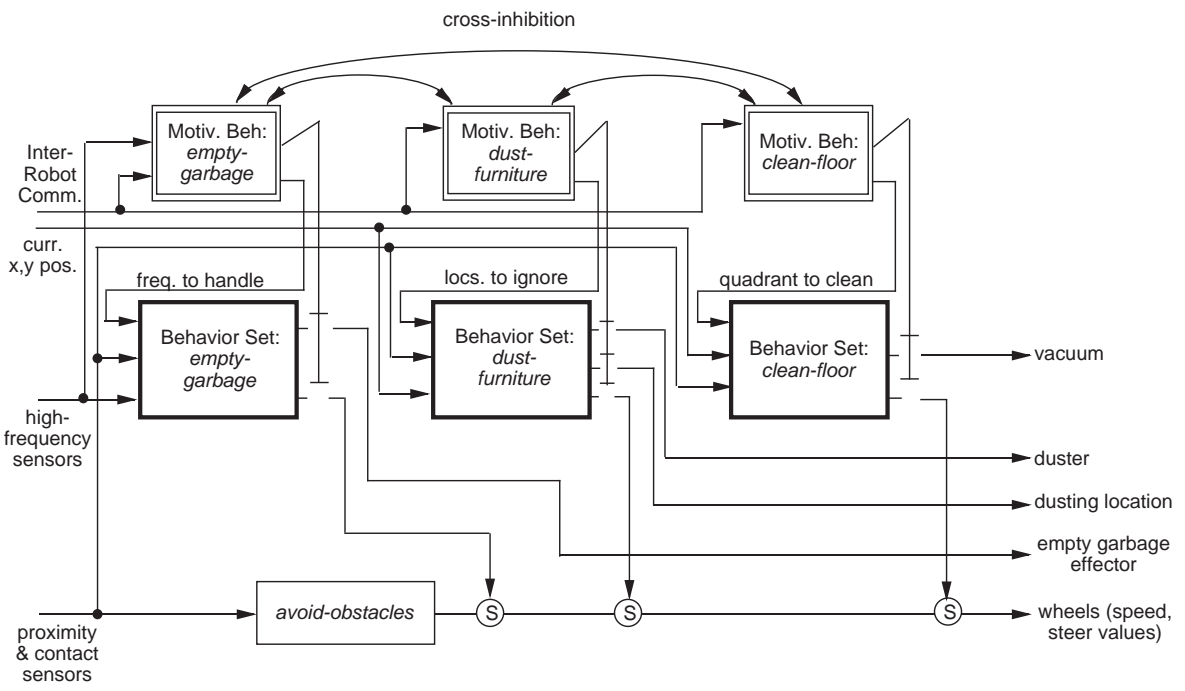

Figure 3: The ALLIANCE-based control for the janitorial service mission. Not all inputs and outputs are shown. Refer to Figures 4 through 6 for more detail.

robot which can perform all of the tasks required in the janitorial service mission. Since the behavior sets are independent, those behavior sets not appropriate for a robot without all of these capabilities can be easily removed from that robot's software control. In this design, as specified by the ALLIANCE approach, the three behavior sets are each controlled by a motivational behavior.

Since the purpose of this implementation is to illustrate the ability of ALLIANCE to generate fault tolerant, adaptive cooperative control, rather than to generate the ideal garbage emptier, furniture duster, or floor cleaner, we made a number of assumptions about the capabilities of the simulated robots and the structure of the robot team's environment. These assumptions are described in the following subsections as the control for each of the three behavior sets is discussed.

Empty-garbage behavior set

The software design of the empty-garbage behavior set is shown in Figure 4. To simplify a robot's detection of the garbage cans for this implementation (since, again, the focus here is on cooperative control rather than on object recognition), we assume that a high frequency emitter is attached to each can. To allow the garbage emptying task to be divided across more than one robot, we assume that a small number of different frequency emitters are available, and are distributed somewhat uniformly across the garbage cans in the room. We 


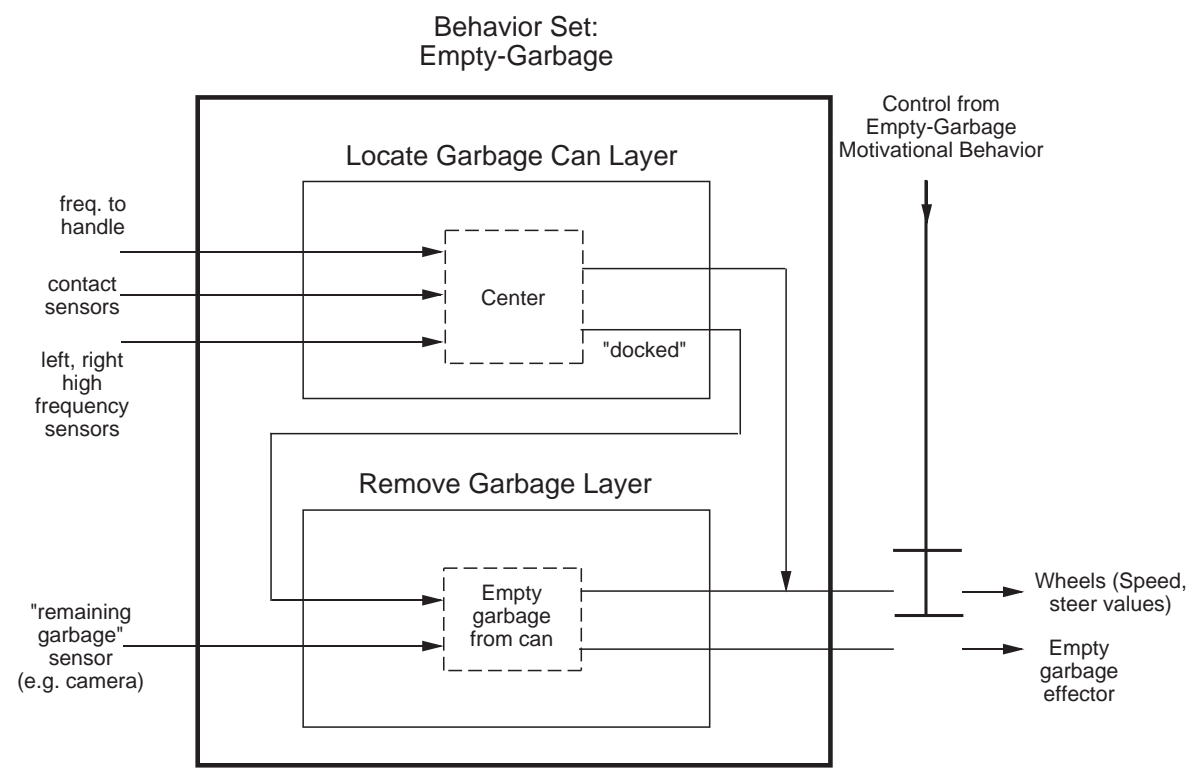

Figure 4: The robot control organization within the empty-garbage behavior set.

further assume that each garbage emptying robot has two sensors for detection of each frequency used in the mission, for use in localization. Using more than one frequency allows one robot to concentrate on emptying the cans emitting a given frequency while some other robot empties those of a different frequency. Finally, we assume that once the garbage is emptied out of a particular can, that garbage can's high frequency emitter is turned off.

The sensory feedback required before the empty-garbage behavior set can be activated is the detection of some high frequency sound which indicates the need to empty a garbage can. Once this sensory feedback is received, the emptygarbage motivational behavior grows impatient to activate its behavior set for that high frequency sound at either a fast or a slow impatience rate, depending upon the current activities of the team members. Note that the motivational behavior monitors more than one rate of impatience for this behavior set one for each pair of frequency detectors of the given robot. If some other robot is currently working on emptying the garbage cans emitting a given frequency (as indicated through its broadcast communication message of the type " $r_{i}$ emptying garbage frequency $f$ "), then the corresponding motivation grows at a slow rate; otherwise, it grows at a fast rate. Once the motivation to activate the behavior set for a given frequency value has grown above threshold, the motivational behavior sends a message to the behavior set indicating the garbage can frequency that behavior set should listen to, and the behavior set becomes active. The behavior set then proceeds to follow the gradient of the 
given frequency until it reaches the garbage can, at which point the effector for emptying the garbage (which could be a powerful vacuum, a manipulator, etc.) is activated until the can is emptied.

Dust-furniture behavior set

The software design of the dust-furniture behavior set is shown in Figure 5. In this mission, a dustable piece of furniture is defined as any object less than a given height. This is implemented on the robots by having two rings of proximity sensors - one higher than the given dustable object height and one lower than the given height. Thus, any object which triggers the second ring of sensors, but not the first ring, should be dusted. To reduce the difficulty of the dusting robot's task, we assume that each furniture dusting robot is told the number of dustable objects in the room at the beginning of the mission (again, the focus here is on cooperative control, rather than object recognition or 3D environmental modeling). However, the robots do not know the locations of the furniture, and must thus search for them. We further assume that some global positioning system (such as [13]), is available that allows robots to uniquely identify a dustable piece of furniture by location. Finally, we assume that when a robot activates the dust-furniture behavior set, it broadcasts one of three messages: (1) if the robot is currently searching for a dustable object, it broadcasts a generic " $r_{i}$ dusting furniture" message; $(2)$ if the robot has actually located a yet-to-be-dusted dustable object, it broadcasts a message such as " $r_{i}$ dusting furniture at location $x, y$ ", which indicates the global position of the piece of furniture it is dusting; or (3) if the robot has concluded the dusting of a piece of furniture, it broadcasts a message such as " $r_{i}$ completed dusting of object at location $x, y$ ".

The sensory feedback required for the dust-furniture motivational behavior to consider activating its behavior set in robot $r_{i}$ is that fewer than the given number of dustable objects have actually been dusted either by $r_{i}$ or by some other robot team member (as indicated through the broadcast communication mechanism). Thus, the dust-furniture motivational behavior becomes impatient at a fast rate of impatience as long as dustable objects remain to be dusted. However, the dust-furniture task is considered to be a task that can be shared by more that one robot team member. Thus, we would like more than one robot to be able to search for objects to dust, but we do not want them to attempt to dust the same object at the same time, or to dust an already-dusted piece of furniture. Thus, the motivational behavior also keeps track of the locations of furniture objects that have been dusted or are being dusted by some other robot. If another robot, $r_{k}$, is currently dusting some object, $o_{j}$, then the motivational behavior of robot $r_{i}$ allows $r_{k}$ some period of time (according to a slow rate of impatience) to complete the dusting of that object. However, if $o_{j}$ remains undusted for a period of time (as indicated by the communication feedback), $r_{i}$ 's dust-furniture motivational behavior becomes impatient with $r_{k}$, and thus $r_{i}$ considers $o_{j}$ as a potential object that it, itself, can dust. The 


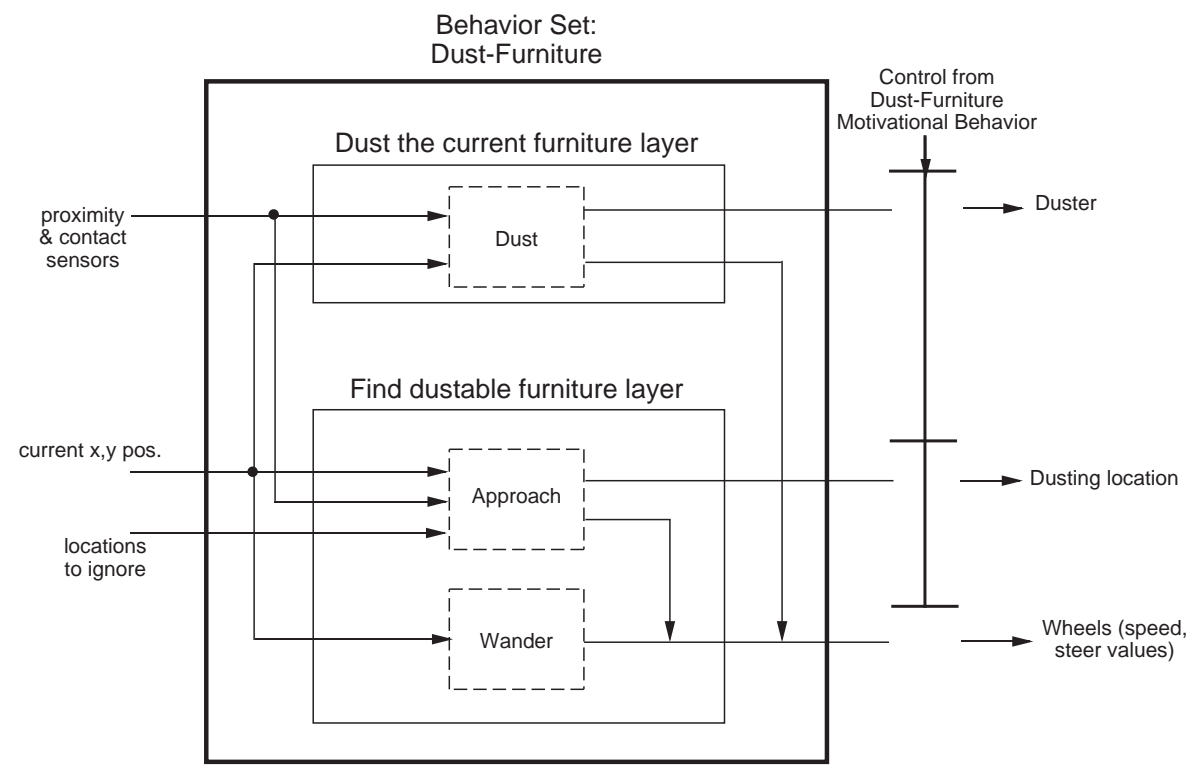

Figure 5: The robot control organization within the dust-furniture behavior set.

information about which dustable objects to ignore is conveyed to the behavior set by the motivational behavior. Thus, whenever the motivation to activate the dust-furniture behavior set crosses the threshold of activation, the motivational behavior also sends the locations to the dust-furniture behavior set of furniture objects to ignore. The dust-furniture behavior set then searches for objects to dust, but ignores those dustable objects which are located at one of the locations on the "ignore list". In this manner, the robots can share the task of dusting the furniture without getting in each other's way.

The dust-furniture behavior set involves a simple wandering technique to search the room for objects yet to be dusted. A more methodical method of searching the room, such as that used in the clean-floor behavior set (see below), could certainly be used instead of a random walk. However, it is interesting to use a variety of techniques in this application to investigate the characteristics of the various approaches. Once a dustable object not on the "ignore list" is located, it is approached, and the robot uses some dust effector to dust the object as it circles around the accessible sides of the object.

Clean-floor behavior set

The third task of the janitorial service team is to clean the floor. The organization of the clean-floor behavior set is given in Figure 6. To ensure that the entire floor gets cleaned efficiently, this behavior set utilizes a coarse grid map to keep track of the areas already cleaned, those yet to be cleaned, and those 


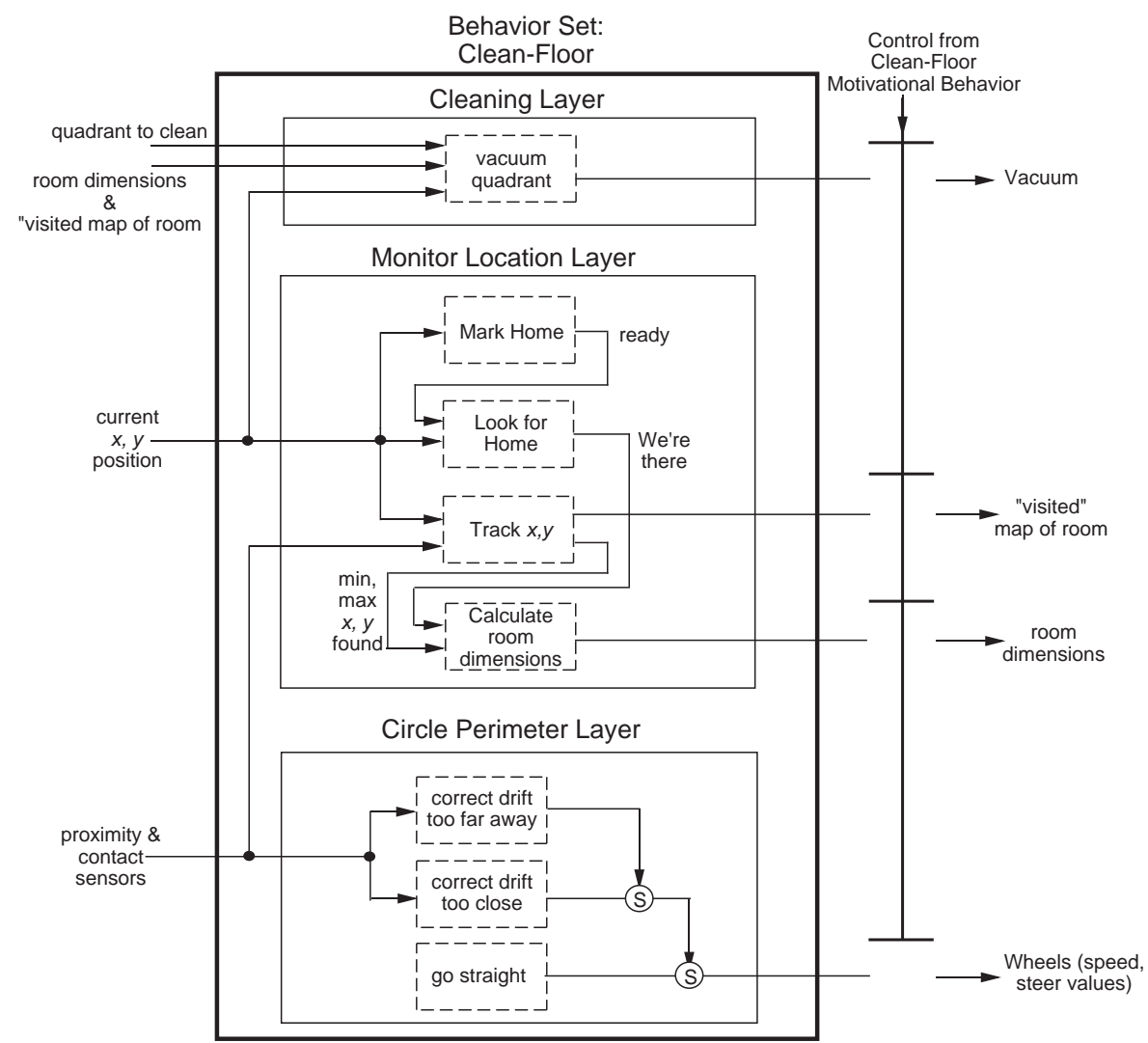

Figure 6: The robot control organization within the clean-floor behavior set.

that are inaccessible due to obstacles. An assumption for this behavior set is that the room to be cleaned is an enclosed area whose shape is unknown. A cleaning robot is then first required to circle the perimeter of the room to determine its shape, cleaning as it goes, and then to begin cleaning the remainder of the room. As with the empty-garbage and dust-furniture tasks, we would like the clean-floor task to be potentially divided across more than one robot. Thus, this behavior set is designed to clean the floor by quadrants after the initial room dimensions are determined, with different robots potentially cleaning different quadrants of the room. The messages communicated by the clean-floor motivational behavior are of three types: (1) " $r_{i}$ finding room dimensions", (2) " $r_{i}$ cleaning quadrant $q$ ", and (3) " $r_{i}$ completed cleaning of quadrant $q$ ".

The sensory feedback required before the clean-floor behavior set can be activated in robot $r_{i}$ is that some quadrant remains to be cleaned either by robot $r_{i}$ or by some other robot, as determined from the broadcast communication 
messages. The motivation to activate the clean-floor behavior set thus grows at a fast rate of impatience as long as some quadrant remains to be cleaned. However, as with the dust-furniture and empty-garbage behavior sets, we do not want robots to interfere with each other's efforts by having all the cleaning robots selecting the same quadrant to clean, to the exclusion of the remaining quadrants. Thus, the motivational behavior keeps track of the quadrants currently being cleaned by other robots, and becomes impatient to clean those quadrants at a slow rate of impatience. When the motivation to activate cleanfloor crosses the threshold of activation, the motivational behavior informs the clean-floor behavior set of the quadrant it should clean, based upon these rates of impatience. When the clean-floor behavior set is activated in robot $r_{i}, r_{i}$ first determines the dimensions of the room as described above (if it has not already found the dimensions), and then begins cleaning the quadrant specified by the motivational behavior.

Cleaning a quadrant requires the robot to visit every square within a coarse grid of that quadrant, or determining through contact and proximity sensors that a grid area is inaccessible due to obstacles. The simplistic algorithm used for covering the quadrant is as follows:

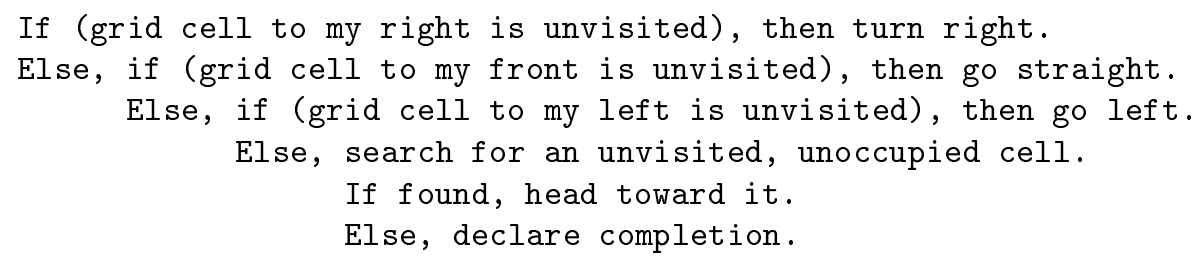

As the robot traverses the quadrant, it uses its proximity and contact sensors to mark grid cells occupied by obstacles. It also marks its current grid location as visited. The coarseness of the grid, the location of obstacles, and the simplistic nature of this traversal algorithm leads to an interesting "snaking" pattern of coverage, as shown in the later snapshots of Figure 7, which is explained in more detail below.

5.2.2. Results of Janitorial Service Implementation. Snapshots of a typical run of the janitorial service robot team simulation are shown in Figure 7. The values of the motivations during this run are shown in Figure 8 . In this run, the team is composed of three robots, which are shown in the lower left corner of the first frame of Figure 7. Each of these robots can perform two of the tasks of the janitorial service mission: robot $r_{1}$ (the leftmost robot) can perform empty-garbage and dust-furniture, robot $r_{2}$ (the center robot) can perform empty-garbage and clean-floor, and robot $r_{3}$ (the rightmost robot) can perform dust-furniture and clean-floor. In this example, the mission involves three garbage cans and three dustable objects; Figure 2 indicates the identity of the various objects in these simulation snapshots.

Under the ALLIANCE control, these robots were able to successfully divide the tasks among themselves in a reactive and dynamic fashion to accomplish 

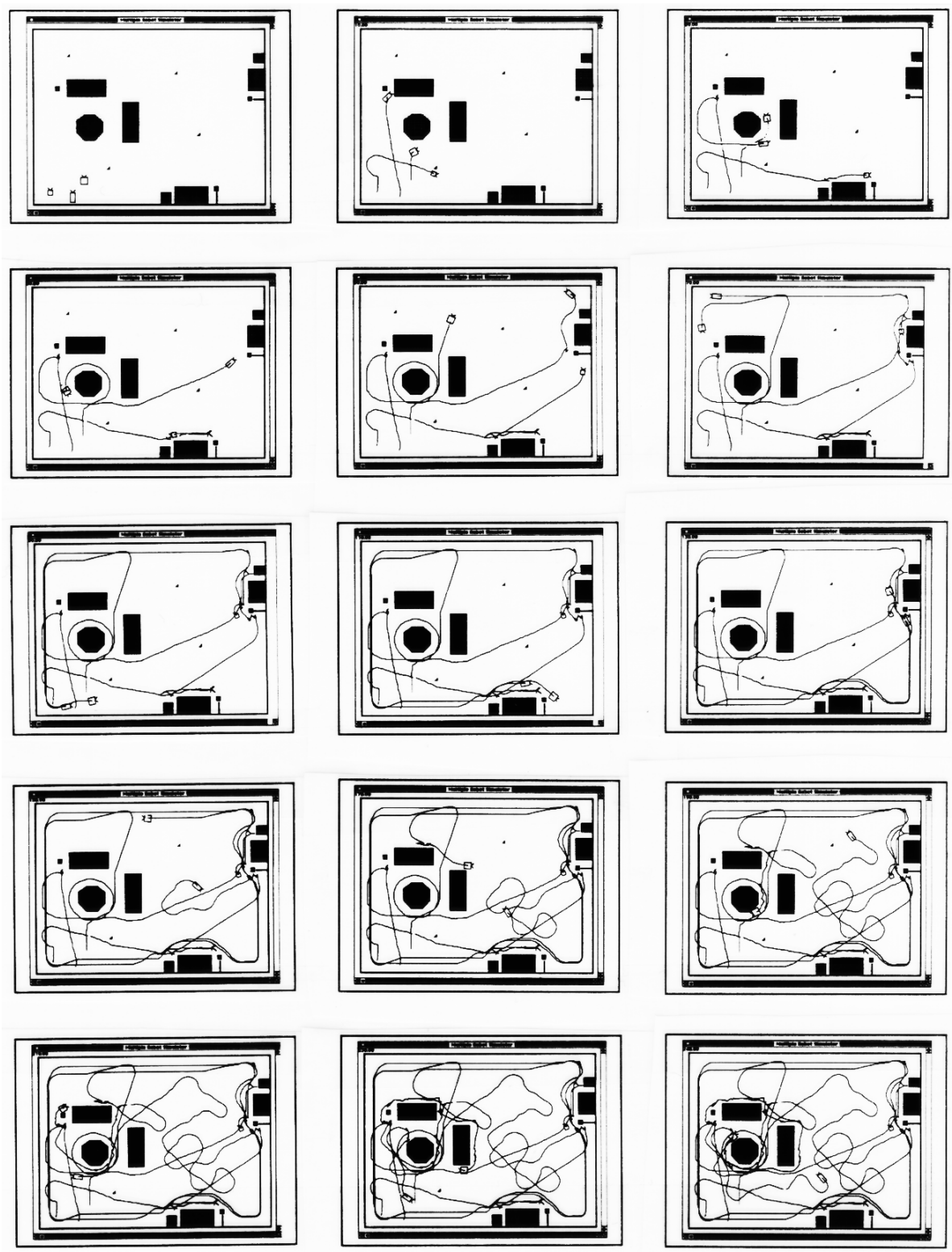

Figure 7: A typical run of the janitorial service simulation (read the snapshots from left to right, top to bottom; robots are numbered $r_{1}, r_{2}, r_{3}$ from left to right in the first snapshot). 

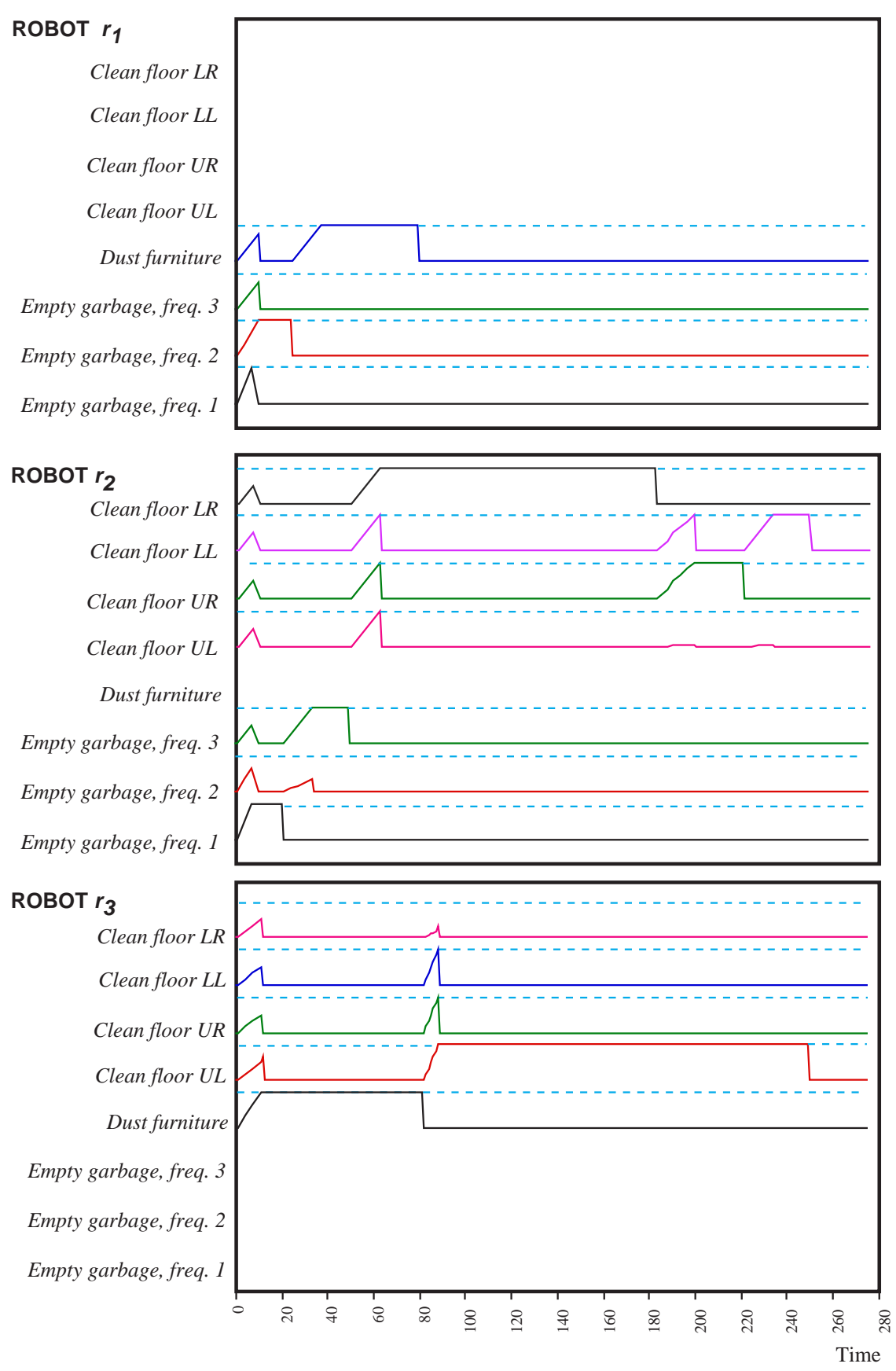

Figure 8: The behavior values during the run of the janitorial service mission shown in Figure 7. The dashed lines above the behavior values indicate the thresholds of behavior activation. 
their mission without duplicating the actions of other robots. As an example of the adaptation of action selection due to the actions of other robots, consider the first 4 frames of Figure 7 . Initially, robots $r_{1}$ and $r_{2}$ both select the action of emptying the garbage, and both head toward the closest garbage can, while robot $r_{3}$ elects to dust the furniture. However, upon hearing that robot $r_{2}$ is also headed to empty the same garbage can, robot $r_{1}$ is satisfied that that garbage can will be emptied, and thus selects another garbage can to empty (in this case, the one to the lower right, as shown in the second snapshot). After emptying its garbage can, robot $r_{2}$ then heads toward the third garbage can to the right center of the room (as seen in the third and fourth snapshots), bypassing the garbage can that robot $r_{1}$ has emptied. Robot $r_{1}$ is then satisfied that all garbage cans will be emptied, even though robot $r_{2}$ has not yet reached the third garbage can, and proceeds to select another action - that of dusting furniture - as seen in the fourth snapshot.

Also shown in the fourth snapshot is robot $r_{2}$ completing the emptying of the last garbage can. Robot $r_{2}$, in snapshot five, then selects to clean the floor, which first requires circling the perimeter of the room, as shown in snapshots five through nine. In the meantime, back in the fifth snapshot, $r_{3}$ completes its dusting of the circular object, and proceeds to search for another object to dust. It wanders the room until the sixth snapshot, at which time it hears that $r_{1}$ has completed dusting the last piece of furniture. This causes $r_{3}$ to go on to another task, namely that of cleaning the floor. To clean the floor, robot $r_{3}$ first circles the perimeter of the room, as shown in snapshots six through ten. It then proceeds to clean the upper left quadrant. (A more efficient implementation would involve a robot informing its teammates of the shape of the room once it is discovered.)

After $r_{1}$ completes its furniture dusting in snapshot six, all of its tasks emptying the garbage and dusting the furniture - are complete. Thus, it has nothing left to do, causing it to wait out the rest of the mission in its current location.

Once $r_{2}$ completes its circle of the perimeter in snapshot nine, it begins cleaning the lower right quadrant in snapshots ten and eleven while robot $r_{3}$ continues its cleaning of the upper left quadrant. Robot $r_{2}$ then goes on to clean the floor of the upper right quadrant in snapshot 12 , and then to the lower left quadrant in snapshots 13 through fifteen. In the meantime, the obstacles in the upper left quadrant have caused $r_{3}$ difficulties in cleaning the upper left quadrant. It finally completes its cleaning of that quadrant in the final snapshot, at which time the mission is complete.

In other experiments with this mission, many unexpected events have been modeled to illustrate the adaptiveness of the architecture to the dynamic environment and the actions of other robots. For example, in the discussion above, if either of the robots $r_{1}$ or $r_{2}$ were unsuccessful in emptying one of the garbage cans, the other robot would become impatient with the lack of progress and proceed to empty that can regardless of the fact that the other robot had se- 
lected that action earlier. If additional garbage cans are added, the robots react fluidly to the environmental change and empty the new cans as if they were always present. If an existing garbage can is suddenly empty, the robots again react immediately by pursuing some other task. New team members can be added to the group and are allowed by the existing team members to help with the mission. Many other such changes to the environment and the abilities of the robots were simulated and handled successfully by this architecture.

\subsection{The Bounding Overwatch Mission}

An additional, quite different, simulation application also implemented using ALLIANCE is the bounding overwatch problem. This mission requires a team of two types of robots to dynamically divide themselves into two subgroups having equal distribution of robot types, and then to travel to the initial assembly points of their respective subgroups and determine a subgroup leader. Next, one team must head out for the next waypoint (i.e., they bound) while the other team monitors their progress and remains alert for danger (i.e., they overwatch). Once the first team reaches its waypoint, the roles of the teams switch, so that the first team overwatches while the second team bounds. This mission is motivated by a military surveillance scenario, in which a team of autonomous vehicles (such as tanks) must safely traverse an area thought to be occupied by enemy forces. This mission illustrates how ALLIANCE can be used for the fault tolerant execution of applications that involve many ordering dependencies among tasks.

Figure 9 shows the ALLIANCE-based control of the robots under the bounding overwatch mission. In this specific implementation, the robots have five behavior sets - join-group, emerge-leader, follow-leader, lead-to-waypoint, and overwatch. Heterogeneity of robots can occur when different robots specialize in different or additional aspects of the mission. For example, robots could specialize in locating safe waypoints, overwatching for danger, or carrying heavy supplies. For any tasks required by the mission that are not shown in Figure 9, additional behavior sets would be added to the robot control.

A typical run of the bounding overwatch mission is shown in Figure 10, with the corresponding motivation values for the uppermost robot team shown in Figure 11. At the beginning of the mission, the only behavior set whose sensory feedback is satisfied is the join-group behavior set. Since this task must be performed by all robot team members, the motivational behaviors in all the robots activate this behavior set at the beginning of the mission. This behavior set is important because it allows the team of robots to divide themselves into two equal subgroups. This division is accomplished using the following simple rule in each robot:

Wait a random length of time $t$ (between 0 and some prespecified maximum time). 


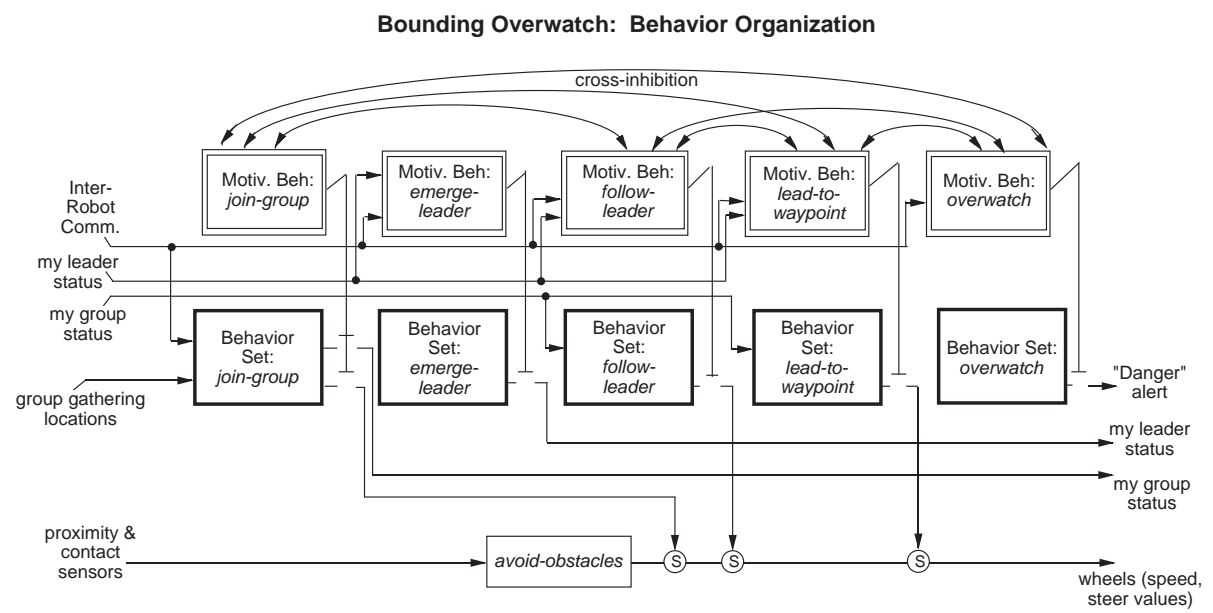

Figure 9: The ALLIANCE-based control for the bounding overwatch mission.

While waiting, monitor the messages of robot team members, keeping track of the number and type of robots in each subgroup so far.

After the random wait period is over, do the following:

1. Select the subgroup with the minimum number of my type so far.

2. If the two subgroups have equal distributions of my type,

Then: Select the group with the fewest members, breaking ties arbitrarily.

3. Broadcast the group I have joined and my robot type.

Once a robot has joined a group, it moves to the prespecified gathering location for its group. The first snapshot of Figure 10 shows the initial location of a group of eight robots - four of each of two types. The second snapshot shows the robots dynamically dividing into the two groups and moving to the specified gathering locations (indicated in Figure 10 by the two small triangles closest to the robot starting locations).

The preconditions for the emerge-leader behavior set to activate in robot $r_{i}$ are that (1) $r_{i}$ has arrived at its group's initial gathering point, and (2) $r_{i}$ 's group does not yet have a group leader. If these conditions are met, then the emergeleader behavior set is activated. The result is that the first robot to arrive at its group's gathering point becomes that group's leader. An interesting side-effect of this definition is that if, at any point in the future, robot $r_{i}$ 's group loses its leader, then $r_{i}$ will become motivated to emerge as the team's leader. Since many other team members will also have this motivation, the relative rates of impatience across robots will determine which robot actually does emerge as the leader. Ideally, the rates of impatience are set such that the robots 

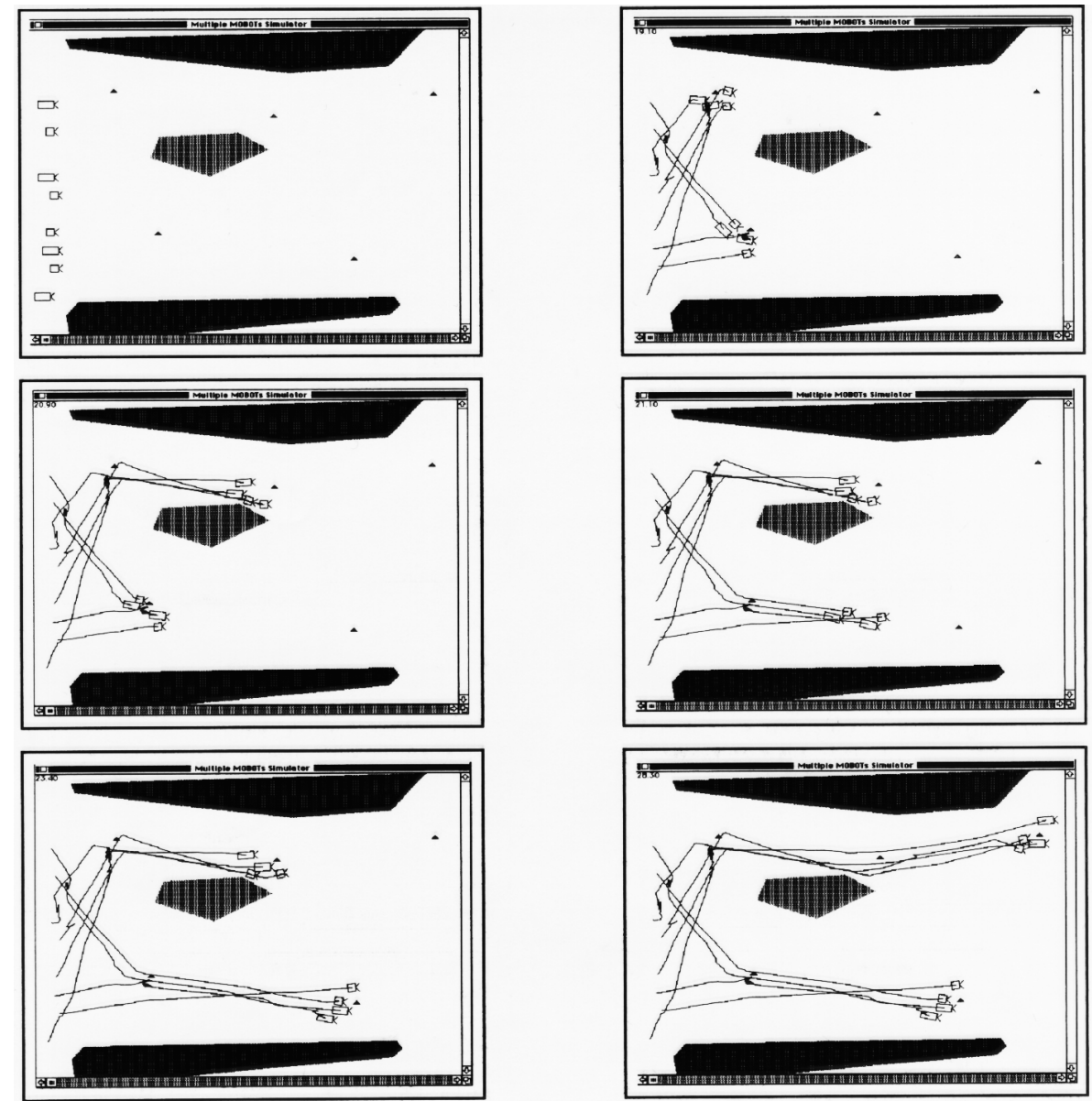

Figure 10: A typical run of the bounding overwatch mission (read the snapshots from left to right, top to bottom). 


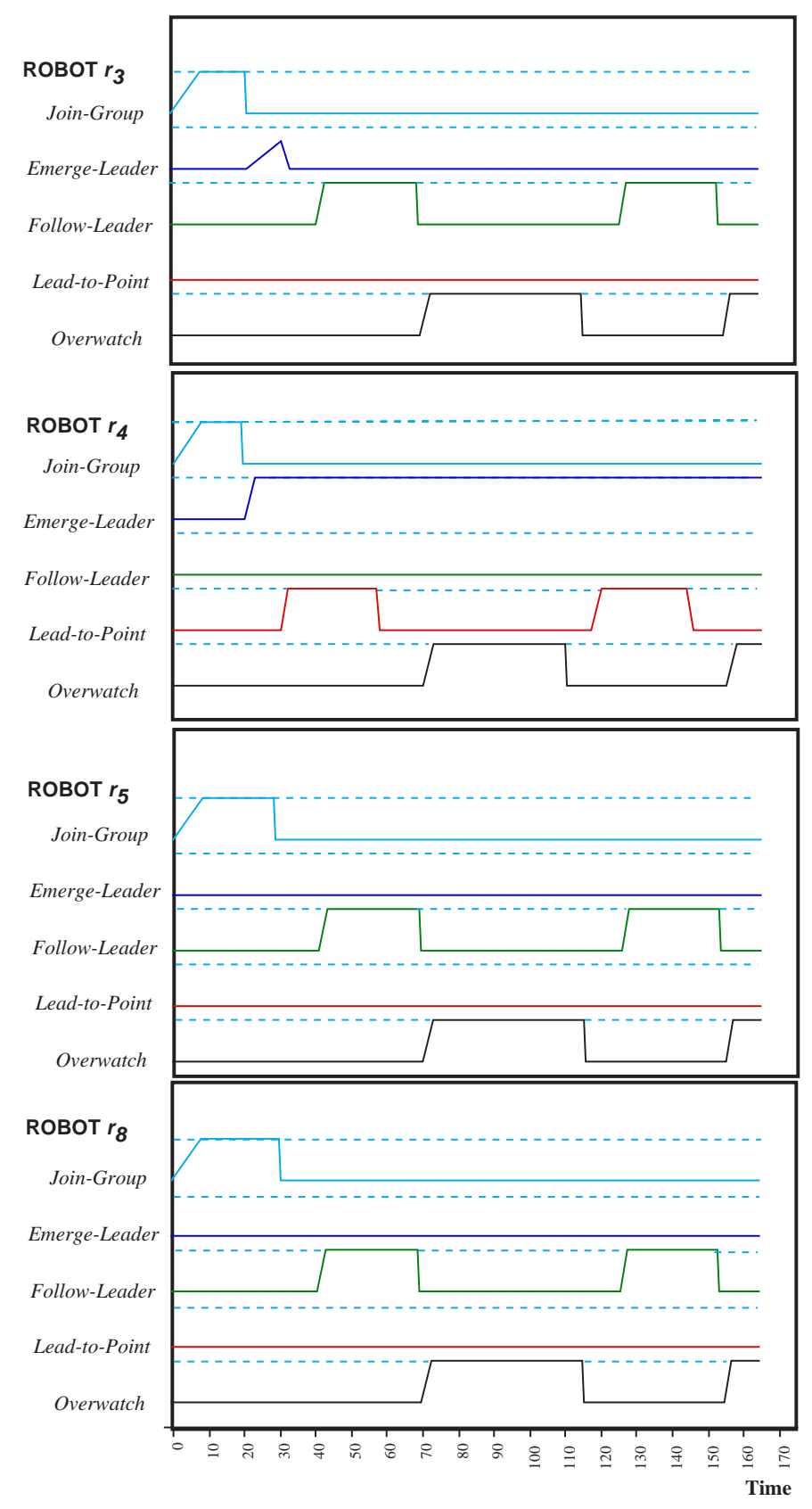

Figure 11: The behavior values during the run of the bounding overwatch mission shown in the previous figure. The dashed lines above the behavior values indicate the thresholds of behavior activation. 
which make better leaders become motivated more quickly. A fixed priority mechanism among the robots breaks ties should they occur. Figure 12 shows the motivations during a run of the bounding overwatch mission in which the leader $\left(r_{4}\right)$ fails, leading to the emergence of robot $r_{3}$ as the new leader.

The precondition for the lead-to-waypoint behavior set in a leader robot is that the previous team has just bounded to its next waypoint. In order to initiate the bounding at the beginning of the mission, this condition is hardcoded into the leader of the first team as soon as its team has collected at the initial gathering location. Thus, the leader of the first team initiates the bounding to the next waypoint. This, in turn, satisfies the preconditions of the follow-leader behavior set in the remaining robots on the first team. The result is that the leader robot leads the team to the next waypoint while the rest of its team follows along. The members of the second team, in the meantime, have activated their overwatch behavior sets, and are overwatching the first team's progress. This scenario is shown in the third frame of Figure 10.

Once the first team's leader has arrived at its next waypoint, the completion of the lead-to-waypoint is broadcast. Upon hearing this, the leader of the second team's preconditions for lead-to-waypoint are satisfied, causing it to lead its team to its next waypoint while the first team overwatches. This continues, as shown in Figure 10, until the teams reach some prespecified destination.

The behavior-based design of the motivational behaviors also allows the robots to adapt to unexpected environmental changes which alter the sensory feedback. The need for additional tasks can suddenly occur, requiring the robots to perform additional work, or existing environmental conditions can disappear and thus relieve the robots of certain tasks. In either case, the motivations fluidly adapt to these situations, causing robots to respond appropriately to the current environmental circumstances. More complex executions of this mission have been performed in simulation that involve numerous additional robot roles (such as clearing paths, monitoring the rear of the group, searching for good waypoints, and so forth) that must be carried out by various team members. These roles can be easily and dynamically reallocated among team members with the ALLIANCE architecture when needed due to the failure of robot team members or due to increased requirements of the mission (perhaps due to an attack of enemy forces.

\section{MEETING DESIGN REQUIREMENTS}

We now review the design requirements outlined in section 2 and examine the extent to which ALLIANCE meets these design goals. Recall that the primary design goal was to develop a cooperative architecture that allowed heterogeneous robots to cooperate to accomplish a mission while exhibiting robustness, reliability, flexibility, and coherence. As these issues are reviewed here, note that the development of a cooperative robot architecture can actually be viewed as the development of an individual robot control architecture that facilitates a 


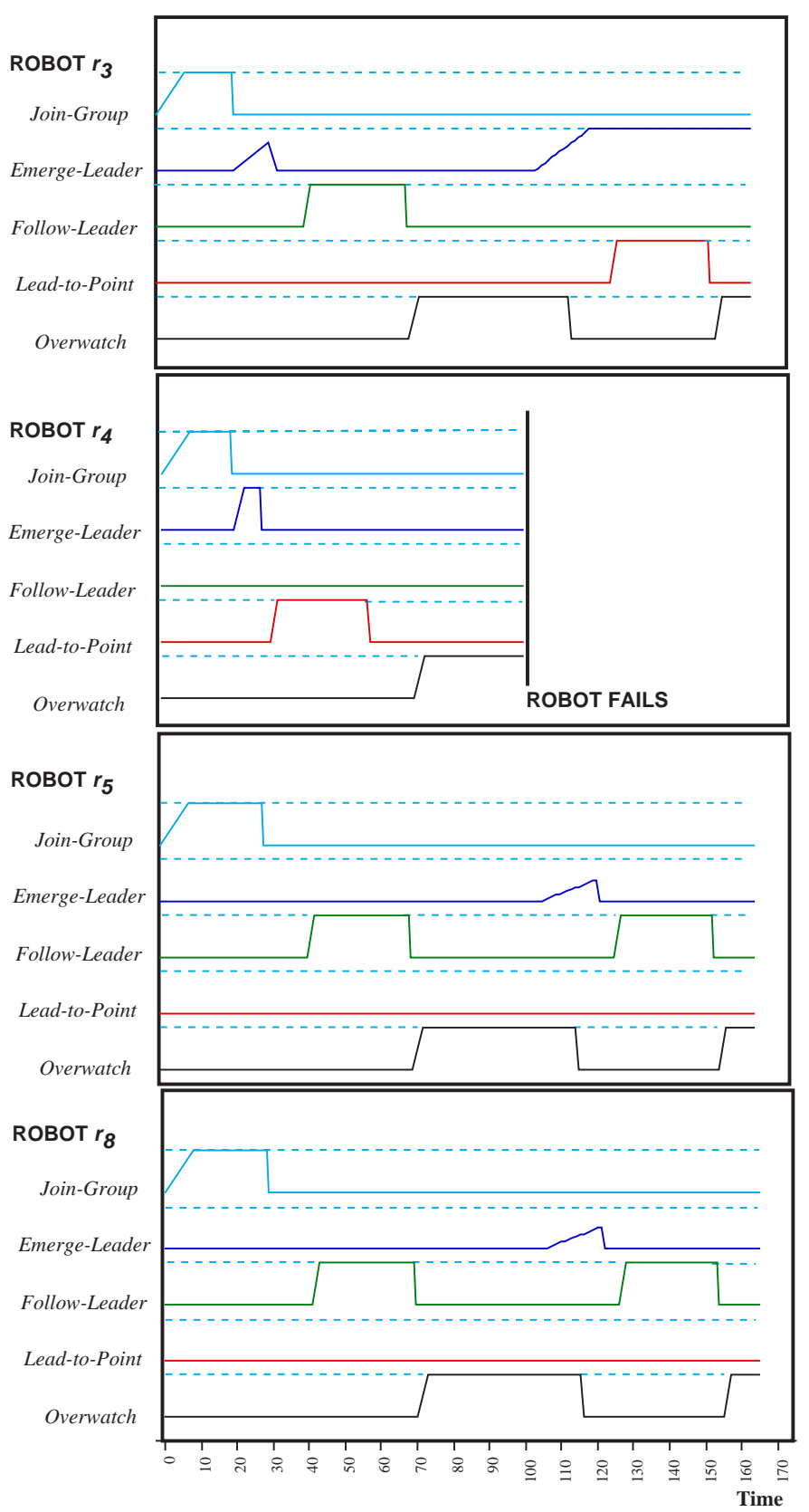

Figure 12: The behavior values during the run of the bounding overwatch mission, in which the original leader $\left(r_{4}\right)$ fails. The dashed lines above the behavior values indicate the thresholds of behavior activation. 
single robot's cooperation with other similarly-designed robots. Thus, we describe how each of these performance issues are addressed in ALLIANCE both from the view of an individual robot control strategy and from the view of a collective team strategy.

\subsection{Robustness and Fault Tolerance}

As described earlier, fault tolerance and robustness refer to the ability of a system to detect and gracefully compensate for partial system failures. In ALLIANCE, each individual robot is designed using a behavior-based approach which ensures that lower levels of competence continue to work even when upper levels break down. In addition, individual robots can be given multiple ways to perform certain tasks, allowing them to explore alternative approaches when met with failure.

From the viewpoint of the team, ALLIANCE first enhances robustness by being fully distributed. Unlike hierarchical architectures, since no individual robot in ALLIANCE is responsible for the control of other robots, the failure of any particular robot is not disproportionally damaging. Secondly, ALLIANCE enhances team robustness by providing mechanisms for robot team members to respond to their own failures or to failures of teammates, leading to a reallocation of tasks (such as the leader role in the bounding overwatch application) to ensure that the mission is completed. Third, ALLIANCE allows the robot team to accomplish its mission even when the communication system breaks down. Although the team's performance in terms of time and energy may deteriorate without communication, at least the team is still able to accomplish its mission. Finally, ALLIANCE enhances team robustness by making it easy for robot team members to deal with the presence of overlapping capabilities on the team. The ease with which redundant robots can be incorporated on the team provides the human team designer the ability to utilize physical redundancy to enhance team robustness.

\subsection{Reliability}

Reliability refers to the dependability of a system and whether it functions properly each time it is utilized. ALLIANCE is designed for applications involving a significant amount of uncertainty in the capabilities of robot team members which themselves operate in dynamic, unpredictable environments. In ALLIANCE, reliability is measured in terms of the architecture's ability to have the robot team accomplish its mission each time the mission is attempted. It can be shown that under certain conditions ALLIANCE is guaranteed to allow the robot team to complete its mission, except when robot failures eliminate required capabilities from the team (from which no architecture could recover). While a rigorous presentation of this proof is beyond the scope of this paper (see [22] for more details), we sketch the proof briefly here. 
It can be shown that ALLIANCE allows teams of robots that have the following characteristics:

- monotonically increasing motivations to perform the tasks of the mission,

- finite thresholds of activation, and

- sufficient task coverage (i.e. for all tasks of the mission, some robot is present that has the ability to perform that task)

to successfully accomplish their mission whenever a condition called Progress when Working is true and when no robots with the sole capability to solve certain tasks of the mission fail. The Progress when Working condition is true when a robot makes progress toward achieving a task when it activates its behavior sets.

The proof of this claim is based upon the allowable values of the motivations of the robots and the possible interactions of robot task reallocations. The allowable values of the motivations lead to the fact that a robot always has a strictly increasing motivation to perform some incomplete task, which in turn leads to the activation of the corresponding behavior set, and thus the completion of a portion of the task. Evaluating the possible interactions of robot task reallocations in terms of impatience and acquiescence values leads to the conclusion that the tasks of the mission must eventually be completed in finite time. The ALLIANCE action selection mechanism thus gives a means for the robot team to achieve its mission reliably when these conditions hold.

\subsection{Flexibility and Adaptivity}

Flexibility and adaptivity refer to the ability of robots to modify their actions as the environment or robot team changes. The motivational behavior mechanism of ALLIANCE constantly monitors the sensory feedback of the tasks that can be performed by an individual agent, adapting the actions selected by that agent to the current environmental feedback and the actions of its teammates (such as the subgroup selection of robots in the bounding overwatch application). Whether the environment changes to require the robots to perform additional tasks or to eliminate the need for certain tasks, ALLIANCE allows the robots to handle the changes fluidly and flexibly. ALLIANCE enhances the adaptivity and flexibility of a robot team by providing mechanisms for robots to work with any other robots designed using ALLIANCE; the robots are not required to possess advance knowledge of the capabilities of the other robots.

One limitation of ALLIANCE is that the parameters of the system (i.e. rates of impatience, acquiescence, and communication) must be provided by the human, thus limiting ALLIANCE's ability to improve its performance over time through adaptivity. An extension to ALLIANCE that we have developed, called L-ALLIANCE [22] (beyond the scope of the current paper) further extends the 
flexibility and adaptivity of the system by allowing robots to learn about their own abilities and the abilities of their teammates in order to improve their performance on subsequent trials of similar missions whenever familiar agents are present.

\subsection{Coherence}

Coherence refers to how well the actions of individual agents combine towards some unifying goal. For individual agents, ALLIANCE causes robots to work only on those tasks which the environmental feedback indicates need to be executed. Thus, ALLIANCE will not cause an individual agent to work on some task that is not required by the mission, nor consistent with the current state of the environment.

Obtaining coherence at the team level requires that robots have some means of determining the actions of other robots and/or the effect of those actions on the environment. Without this knowledge, the robots become a collection of individuals pursuing their own goals in an environment that happens to contain other such robots. While we certainly want the robots to be able to accomplish something useful even without knowledge of other robots on the team, ideally each robot should take into account the actions of other robots in selecting their own actions.

Determining the actions of other robots can be accomplished through either passive observation or via explicit communication. Since passive action recognition is very difficult and is a major research topic in itself, ALLIANCE augments the observation skills of the robot team members through the use of one-way broadcast communication that provides each robot with an awareness of the actions of other robots, plus the ability to act on that information. With this awareness, robots do not replicate the actions of other robots, thus giving them more coherence. We note the importance of this mechanism to achieve team coherence, since when the communications mechanism is unavailable, team coherence is reduced. Refer to [23] for a more detailed discussion of this issue.

Note that a number of issues regarding the efficiency of ALLIANCE are not addressed here. Among these issues include questions of how long robots remain idle before activating a task, how to ensure that robots failing at one task go on to attempt another task they might be able to accomplish, how robots deal with having more than one way to accomplish a task, and so forth. These issues are addressed in the L-ALLIANCE extension to ALLIANCE [22] through the use of dynamic parameter update mechanisms.

\section{CONCLUSIONS}

In this paper, we have addressed the issue of developing robotics technologies that successfully deal with the dynamics and complexity found in real-world applications. We described the design goals of real-world robotics applications, 
and presented a general architecture - called ALLIANCE - that facilitates the fault-tolerant, adaptive control of small- to medium-sized teams of cooperating robots. The key characteristics of this architecture can summarized as follows:

- Fully distributed (at both the individual robot level and at the team level)

- Applicable to robot teams having any degree of heterogeneity

- Uses one-way broadcast communication; no negotiation or two-way conversations are utilized

- Recovers from failures in individual robots or in the communication system

- Allows new robots to be added to the team at any time

- Allows adaptive action selection in dynamic environments

- Eliminates replication of effort when communication is available

- Provably terminates for a large class of applications

We illustrated the generality of this architecture by presenting two proof-ofconcept implementations: the janitorial service and the bounding overwatch missions. The janitorial service application offers an example of a mission involving numerous independent tasks that must be carried out, and the ability of ALLIANCE to allow robot team members to select their actions to eliminate duplication of effort. The bounding overwatch mission offers an illustration of how several tasks with fixed ordering constraints can be solved using ALLIANCE. This architecture offers an easy way to achieve dynamic role transferral in missions involving changes in the environment or in the robot team. These applications, along with the hazardous waste cleanup mission described in $[20,25]$ illustrate the wide variety of applications for which the ALLIANCE architecture is well-suited.

\section{ACKNOWLEDGEMENTS}

Many thanks to the two anonymous reviewers who offered excellent suggestions on ways to improve this paper. This research was funded in part by the Advanced Research Projects Agency of the Department of Defense under Office of Naval Research contract N00014-91-J-4038 at the Massachusetts Institute of Technology's Artificial Intelligence Laboratory, and in part by the Office of Engineering Research, Basic Energy Sciences (directed by Dr. Oscar Manley), of the U.S. Department of Energy, under contract No. DE-AC05-84OR21400 with Martin Marietta Energy Systems, Inc.

\section{REFERENCES}


1. Ronald C. Arkin, Tucker Balch, and Elizabeth Nitz. Communication of behavioral state in multi-agent retrieval tasks. In: Proceedings of the International Conference on Robotics and Automation. 588-594. (1993).

2. H. Asama, K. Ozaki, A. Matsumoto, Y. Ishida, and I. Endo. Development of task assignment system using communication for multiple autonomous robots. Journal of Robotics and Mechatronics 4, No 2 122-127. (1992).

3. Gerardo Beni and Jing Wang. On cyclic cellular robotic systems. In: Japan - USA Symposium on Flexible Automation. 1077-1083. (1990).

4. (Alan Bond and Less Gasser., Eds.). Readings in Distributed Artificial Intelligence. Morgan Kaufmann. (1988).

5. Rodney A. Brooks. A robust layered control system for a mobile robot. IEEE Journal of Robotics and Automation RA-2, No 1 14-23. (1986).

6. Rodney A. Brooks. Elephants don't play chess. Robotics and Autonomous Systems No 6 3-15. (1990).

7. Philippe Caloud, Wonyun Choi, Jean-Claude Latombe, Claude Le Pape, and Mark Yim. Indoor automation with many mobile robots. Proceedings of the IEEE International Workshop on Intelligent Robots and Systems. 67-72. (1990).

8. Paul Cohen, Michael Greenberg, David Hart, and Adele Howe. Real-time problem solving in the Phoenix environment. COINS Technical Report 9028, U. of Mass., Amherst. (1990).

9. J. Deneubourg, S. Goss, G. Sandini, F. Ferrari, and P. Dario. Self-organizing collection and transport of objects in unpredictable environments. JapanU.S.A. Symposium on Flexible Automation. 1093-1098. (1990).

10. Alexis Drogoul and Jacques Ferber. From Tom Thumb to the Dockers: Some experiments with foraging robots. Proceedings of the Second International Conference on Simulation of Adaptive Behavior. 451-459. (1992).

11. T. Fukuda, S. Nakagawa, Y. Kawauchi, and M. Buss. Self organizing robots based on cell structures - CEBOT. Proceedings of 1988 IEEE International Workshop on Intelligent Robots and Systems (IROS '88). 145-150. (1988).

12. S. Ichikawa and F. Hara. An experimental realization of cooperative behavior in multi-robot system. (H. Asama, T. Fukuda, T. Arai, and I. Endo, Eds.). In: Distributed Autonomous Robotic Systems. Springer-Verlag. (1994).

13. MTI Research Inc. Conac 3-D tracking system. Operating manual. (1995).

14. C. Ronald Kube and Hong Zhang. Collective robotic intelligence. Proceedings of the Second International Workshop on Simulation of Adaptive 
Behavior. 460-468. (1992).

15. T. C. Lueth and T. Laengle. Fault-tolerance and error recovery in an autonomous robot with distributed controlled components. (H. Asama, T. Fukuda, T. Arai, and I. Endo, Eds.). Distributed Autonomous Robotic Systems. Springer-Verlag. (1994).

16. Maja Mataric. Designing emergent behaviors: From local interactions to collective intelligence. Proc. of the Second Int'l Conf. on Simulation of Adaptive Behavior. 432-441. MIT Press. (1992).

17. Fabrice R. Noreils. Toward a robot architecture integrating cooperation between mobile robots: Application to indoor environment. The International Journal of Robotics Research 12, No 1 79-98. (1993).

18. J. Ota, T. Arai, and Y. Yokogawa. Distributed strategy-making method in multiple mobile robot system. Distributed Autonomous Robotic Systems. Springer-Verlag. (H. Asama, T. Fukuda, T. Arai, and I. Endo, Eds.). (1994).

19. Lynne E. Parker. ALLIANCE: An architecture for fault tolerant, cooperative control of heterogeneous mobile robots. Proc. of the 1994 IEEE/RSJ/GI Int'l Conf. on Intelligent Robots and Systems (IROS '94). 776-783. (1994).

20. Lynne E. Parker. An experiment in mobile robotic cooperation. Proceedings of the ASCE Specialty Conference on Robotics for Challenging Environments. (1994).

21. Lynne E. Parker. Fault tolerant multi-robot cooperation. MIT Artificial Intelligence Lab Videotape AIV-9. (1994).

22. Lynne E. Parker. Heterogeneous Multi-Robot Cooperation.. PhD thesis, Massachusetts Institute of Technology, Artificial Intelligence Laboratory, Cambridge, MA, MIT-AI-TR 1465. (1994).

23. Lynne E. Parker. The effect of action recognition and robot awareness in cooperative robotic teams. Proceedings of the 1995 IEEE/RSJ International Conference on Intelligent Robots and Systems (IROS '95). (1995).

24. Lynne E. Parker. L-ALLIANCE: A mechanisms for adaption action selection in heterogeneous multi-robot teams. Technical Report ORNL/TM13000, Oak Ridge National Laboratory. (1995).

25. Lynne E. Parker. ALLIANCE: An architecture for fault tolerant multirobot cooperation. Technical Report ORNL/TM-12920, Oak Ridge National Laboratory. (1995). to appear (with revisions) in IEEE Transactions on Robotics and Automation

26. Luc Steels. Cooperation between distributed agents through self-organization. (Yves Demazeau and Jean-Pierre Muller, Eds.). Decentralized A.I.. Else- 
vier Science. (1990).

27. Daniel Stilwell and John Bay. Toward the development of a material transport system using swarms of ant-like robots. Proceedings of IEEE International Conference on Robotics and Automation. 766-771. (1993).

28. Guy Theraulaz, Simon Goss, Jacques Gervet, and Jean-Louis Deneubourg. Task differentiation in Polistes wasp colonies: a model for self-organizing groups of robots. Proceedings of the First International Conference on Simulation of Adaptive Behavior. 346-355. (1990).

29. Jing Wang. DRS operating primitives based on distributed mutual exclusion. Proceedings of the IEEE/RSJ International Conference on Intelligent Robots and Systems. 1085-1090. (1993).

30. Masaki Yamamoto. Sozzy: A hormone-driven autonomous vacuum cleaner. Proceedings of the SPIE Mobile Robots VIII Conference. (1993). 